\title{
INFORMATION FLOW ANALYSIS FOR FILE SYSTEMS AND DATABASES USING LABELS
}

\author{
Ehud Gudes, Luigi V. Mancini, and Francesco Parisi-Presicce
}

\begin{abstract}
The control of information flow has been used to address problems concerning the privacy and the secrecy of data. A model based on decentralized labels extends traditional multilevel security models by allowing users to declassify information in a distributed way. We extend this decentralized labels model developed by other authors by addressing specific issues that arise in accessing files and databases and in general in I/O operations. While retaining the support for static analysis, we also include run-time checks to allow declassification with "controlled information leakage".
\end{abstract}

\section{INTRODUCTION}

One of the ways used to address the problem of privacy and secrecy of data has been through the use of models for a precise control of the propagation of information [1-3]. Several information flow models have been developed over the years, mostly for traditional multilevel systems [11]. Some of the more recent ones are geared toward object-oriented database systems [9, 10], and toward a decentralized control of access information for a distributed environment [6]. Our work is based on ideas of [5] and [6] and focuses on alternatives to that notion of declassification, especially as it relates to flies and databases.

The decentralized label model of [5] is more complex than traditional models used in classical multilevel security policy. A decentralized label reflects the flow policies of the individual principals on the data so labeled, and the use of the labels is such that the different policies are satisfied simultaneously. Confidentiality is then protected even in the context where the principals distrust each other or their respective code. Note that policy here refers to a single data entity and not to the behavior of the system as a whole. This decentralized label model is based on annotated program code that is statically analyzed. It provides a set of rules that a program must follow to prevent leakage of information. A compiler statically checks the flows of information in a program and allows its execution if all the re-labeling in the program are safe, i.e., legal according to the rules. In traditional information flow control models, declas- 
sification of information is outside the model and is performed by a trusted subject. This aspect makes such models unsuitable in a decentralized environment. In the decentralized label model of [5, 6], limited forms of declassification are allowed to be included explicitly by the programmer, provided that other principals' policies are not affected.

Our objective is to relax some of the restrictions and consider I/O channels, files and databases at run-time, and restricted forms of controlled information leakage.

If the program is compiled when the files to be used at run-time are known, no later check is needed. If the files or the file labels change, it is necessary to recompile the program to verify that the rules for declassification are followed. An alternative to this approach is to use the compilation to produce constraints on the labels of the files to be used: at run-time, there is a check of permissions to verify that the labels of the files actually used satisfy the compile-time constraints. If the constraint is, for example, the requirement that the label of the output file be no less restrictive than the label of the output variables of the program, the problem reduces to the safe relabeling in [6]. But the constraints can be of a more general nature (see Section 3) reflecting a more relaxed policy for information leakage. All this is done independently of the subject executing the program. The security level of the subject executing a program should be taken into account, especially when dealing with I/O channels. This is discussed in this paper.

Declassification can occur at two levels. By the use of the appropriate instruction, declassification takes place inside the program and deals with the labels of variables [5]. The external modification of the label of a file also may constitute a declassification of the information in the file, if for example one of the owners of the file adds a reader to his own policy. This kind of declassification takes place in the administration of files.

Myers and Liskov incorporated the approach and the ideas of $[6,5]$ into the specific programming language framework of Java, and called their extended Java language Jflow [4]. This extended language allows both compile-time and run-time label checking, supports the definition of label data types, and provides for automatic label inference.

Samarati et al. presented a related model in the context of Object-oriented databases [9]. The model provides only for run-time checking by accumulating the flow during program execution. It checks all the flows between objects and between calling and called methods. The checks are done by a special trusted system component called the Message filter. The model was later extended in [10] to support exceptions. In a recent paper [12] Chen, Wijesekera, and Jajodia show how different flow models like Denning's lattice model, and Myers distributed label model can be formalized using a single Prolog-like language Flexflow. A different approach to flow control in a web environment 
was suggested in [13]. In this approach, the flow is controlled by a special encryption based web viewer, but the flow policies supported are quite limited.

In the rest of this paper, Section 2 overviews the basic notions of the model based on distributed control via labels and policies in $[5,6]$ and proposes a relaxation of this model. Section 3 extends the use of the labels to files and I/O operations, and is also concerned with the declassification process, before and after compilation. Section 4 deals with database access and update with views and labels. Section 5 contains a summary and points to future work.

\section{THE LABEL MODEL}

In this section, we briefly describe a label model very similar to the one proposed in [5]. The presentation is slightly different.

The model is based on a notion of label that is used to categorize different entities and to control the transfer of information between two such entities. A label can be viewed as a type and rules are described to define compatible types. We first present a simple version with no relation among the principals, and then we extend it to consider a hierarchy based on the notion of acts for.

Labels are associated with values (computed or read by a program), variables (declared and modified in a program), I/O channels (used by programs), files and views of a database. The compatibility between types determines, for example, whether a variable can hold a value or can be used to read a file, or whether a value can be written into a file or into a database.

Given a set of owners and a set of readers, not necessarily distinct, a label is just a set of pairs $(o, r)$ with $o$ an owner and $r$ a reader. A pair $(o, r)$ in the label $L$ indicates the willingness on the part of the owner $o$ to grant reading rights to $r$ for any information labeled $L$. If there are several owners in the label, the access to information labeled $L$ depends on the combination of the different authorizations.

For a label $L$

$$
\begin{aligned}
& \text { owners }(L)=\{o: \exists \text { reader } r \text { such that }(o, r) \in L\} \\
& \text { readers }(L, o)=\{r:(o, r) \in L\}
\end{aligned}
$$

Moreover, the following set definitions will be useful in the rest of this paper:

$$
\begin{aligned}
& I N T(L)=\bigcap\{\text { readers }(L, o): o \in \text { owners }(L)\} \\
& U N(L)=\bigcup\{\text { readers }(L, o): o \in \text { owners }(L)\}
\end{aligned}
$$

Note that $I N T(L)$ is what in [6] (without the principal hierarchy) is called effective-readers $(L)$.

A label is completely characterized by owners $(L)$ and readers $(L, o)$ for each $o \in$ owners $(L)$.

If owners $(L)=\left\{o_{1}, o_{2}, o_{3}\right\}$, readers $\left(L, o_{1}\right)=\operatorname{readers}\left(L, o_{2}\right)=\left\{r_{1}, r_{2}\right\}$ and readers $\left(L, o_{3}\right)=\left\{r_{1}, r_{3}\right\}$, then it is convenient to denote $L$ by $\left\{o_{1}\right.$ : 
$\left.r_{1}, r_{2} ; o_{2}: r_{1}, r_{2} ; o_{3}: r_{1}, r_{3}\right\}$ as in $[5,6]$ rather than by $\left\{\left(o_{1}, r_{1}\right),\left(o_{1}, r_{2}\right)\right.$, $\left.\left(o_{2}, r_{1}\right),\left(o_{2}, r_{2}\right),\left(o_{3}, r_{1}\right),\left(o_{3}, r_{3}\right)\right\}$.

Based on the above conservative definition of access, there are two ways to declassify information through a modification of the label: either by reducing the set owners $(L)$, that is by removing an owner (more precisely, by removing all the pairs with the specific owner as first component) or by adding rights by augmenting one of the sets readers $(L, o)$ (more precisely, by adding new pairs $(o, r)$ for an existing $\boldsymbol{o})$. For example, a data $x$ labeled $\left\{o_{1}: r_{1}, r_{2} ; o_{2}: r_{1}, r_{2} ; o_{3}: r_{1}, r_{3}\right\}$ can be declassified to allow $r_{2}$ to access it either by removing $\left(o_{3}, r_{1}\right),\left(o_{3}, r_{3}\right)$ (i.e. the entire $o_{3}$ entry), or by adding $\left(o_{3}, r_{2}\right)$. In both cases $r_{2}$ becomes part of the intersection of the readers set of $L$ and is authorized to access $x$ according to the most conservative policy mentioned above.

Labels can be 'ordered' by defining $L_{1} \sqsubseteq L_{2}$ if $L_{2}$ is at least as restrictive as $L_{1}$ in granting permissions. More formally:

DEFINITION 1 (SIMPLE ORDERING) $L_{1} \quad \sqsubseteq_{s} \quad L_{2}$ if and only if owners $\left(L_{1}\right) \subseteq$ owners $\left(L_{2}\right)$ and $\forall o \in$ owners $\left(L_{1}\right)$, readers $\left(L_{2}, o\right) \subseteq$ readers $\left(L_{1}, o\right)$.

A simple fact that follows from the above definition is:

PRoposition 2 (LABELS INTERSECTION) If $L_{1} \subseteq_{s} L_{2}$ then INT $\left(L_{2}\right) \subseteq$ $\operatorname{INT}\left(L_{1}\right)$

\subsection{Ordered owners and readers}

To allow indirect access rights based on inheritance, owners and readers are organized in a principal hierarchy, i.e., structured using a reflexive and transitive relation $\rho$.

A pair $\left(o_{1}, o_{2}\right) \in \rho$ indicates that the owner $o_{1}$ can prevent the owner $o_{2}$ from assigning access rights; a pair $\left(r_{2}, r_{1}\right) \in \rho$ indicates that $r_{1}$ can read whatever $r_{2}$ can read. This hierarchy determines, for each label $L$, a set $E(L)$ of effective flows $(o, r)$ obtained by adding to $L$ all pairs $(o, r)$ implicitly defined (via $\rho$ ) by $L$. To this extent, we define a function $A D D_{\rho}$ on sets $S$ of pairs $(o, r)$ by letting:

$A D D_{\rho}(S)=\left\{(o, r): \exists o^{\prime}\right.$ with $\left.\left(o^{\prime}, o\right) \in \rho,\left(o^{\prime}, r\right) \in S\right\} \cup\{(o, r)$ : $\exists r^{\prime}$ with $\left.\left(r^{\prime}, r\right) \in \rho,\left(o, r^{\prime}\right) \in S\right\}$

Since $\rho$ is reflexive, $S \subseteq A D D_{\rho}(S)$. As a function of $S, A D D_{\rho}(S)$ is monotonic with respect to the Simple Label Ordering, i.e., if $S \sqsubseteq_{s} S^{\prime}$ then $A D D_{\rho}(S) \sqsubseteq_{s} A D D_{\rho}\left(S^{\prime}\right)$. The function $A D D_{\rho}(S)$ is also monotonic in $\rho$ (i.e., if $\rho \subseteq \sigma$, then $A D D_{\rho}(S) \subseteq A D D_{\sigma}(S)$ ), but this fact will not be used here. 
Let us denote $S$ by $A D D_{\rho}^{0}(S)$ and $A D D_{\rho}^{i+1}(S)=A D D_{\rho}\left(A D D_{\rho}^{i}(S)\right)$. Since the sets of owners and readers are finite, the set $\left\{A D D_{\rho}^{i}(S): i \geq 0\right\}$ is finite, with $A D D_{\rho}^{k}(S)$ its largest element for some $k$. The closure of $S$ with respect to $\rho$, denoted by $C l_{\rho}(S)$, is by definition this $A D D_{\rho}^{k}(S)$. We denote the set of readers defined by the above closure as the effective set of readers.

Definition 1 considers all owners and readers unrelated in the hierarchy. By taking into account the principal hierarchy $\rho$ for owners and readers, the definition extends to:

DEFINITION 3 (HIERARCHICAL LABELS ORDERING) $L_{1} \sqsubseteq L_{2}$ if and only if $\forall o_{1} \in$ owners $\left(L_{1}\right), \exists o_{2} \in$ owners $\left(L_{2}\right)$ such that $\left(o_{2}, o_{1}\right) \in \rho$ and $\forall r_{2} \in$ readers $\left(L_{2}, o_{2}\right), \exists r_{1} \in$ readers $\left(L_{1}, o_{1}\right)$ such that $\left(r_{2}, r_{1}\right) \in \rho$.

It is straightforward to verify that $\sqsubseteq$ is reflexive and transitive. If $L_{1} \sqsubseteq L_{2}$ and $L_{2} \sqsubseteq L_{1}$, then $C l_{\rho}\left(L_{1}\right)=C l_{\rho}\left(L_{2}\right)$ but it is not necessarily the case that $L_{1}=L_{2}$.

EXAMPLE 4 The set of owners consists only of $\alpha_{1}$ and $o_{2}$, unrelated by $\rho$, and the set of readers is $\left\{r_{1}, r_{2}, r_{3}, r_{4}\right\}$ with $\left(r_{3}, r_{1}\right) \in \rho$ and $\left(r_{4}, r_{2}\right) \in \rho$. Define $L_{1}=\left\{o_{1}: r_{1} ; o_{2}: r_{2}, r_{4}\right\}$ and $L_{2}=\left\{o_{1}: r_{1}, r_{3} ; o_{2}: r_{2}\right\}$. Then, by definition, $L_{2} \sqsubseteq L_{1}$ since owners $\left(L_{1}\right)=$ owners $\left(L_{2}\right), \rho$ is reflexive and $\left(r_{4}, r_{2}\right) \in \rho$. Similarly $L_{1} \sqsubseteq L_{2}$ using $\left(r_{3}, r_{1}\right) \in \rho$. Furthermore, $C l_{\rho}\left(L_{1}\right)=C l_{\rho}\left(L_{2}\right)=$ $\left\{o_{1}: r_{1}, r_{3} ; o_{2}: r_{2}, r_{4}\right\}$. Obviously, $L_{1} \neq L_{2}$.

Note that taking the hierarchy into account, $C l_{\rho}(L)$ is a simpler way of defining what is called $\mathbf{X} L(L, \rho)$ in [6].

\section{PROPOSITION 5 (CLOSURES ) If $L_{1} \sqsubseteq L_{2}$ then $C l_{\rho}\left(L_{1}\right) \sqsubseteq C l_{\rho}\left(L_{2}\right)$}

The intuition behind $L_{1} \sqsubseteq L_{2}$ is that entities labeled $L_{1}$ can be safely relabeled $L_{2}$, safely in the sense that under the new label $L_{2}$, the number of readers with access to the information is not increased.

So the process of declassifying information consists of re-labeling the information from a label $L_{2}$ to a label $L_{1} \sqsubseteq L_{2}$. Note that the declassification could only be apparent and not real, since we may have $C l_{p}\left(L_{1}\right)=C l_{\rho}\left(L_{2}\right)$ even if $L_{1} \sqsubseteq L_{2}$ and $L_{1} \neq L_{2}$.

We argue, and will discuss it in the next section, that in dealing with files and I/O operations, $L_{1} \sqsubseteq L_{2}$ may be too restrictive as a notion of safe flow and could be relaxed. The confidentiality of the information labeled with $L_{1}$ is preserved by re-labeling it with $L_{2}$ also if $U N\left(L_{2}\right) \subseteq I N T\left(C l_{\rho}\left(L_{1}\right)\right)$, that is if any potential effective reader in any policy in $L_{2}$ is considered trusted (i.e., contained in the set of effective readers) in every policy of $L_{1}$. 
Definition 6 (SAFE RELABEling) An item labeled $L_{1}$ can safely be relabeled $L_{2}$ if either

- $L_{1} \sqsubseteq L_{2}$ or

- $U N\left(L_{2}\right) \subseteq I N T\left(C l_{\rho}\left(L_{1}\right)\right)$

The constraint $U N\left(L_{2}\right) \subseteq I N T\left(C l_{\rho}\left(L_{1}\right)\right)$ is neither more restrictive nor more relaxing than $L_{1} \sqsubseteq L_{2}$ as the next two examples show. So the relaxation consists of requiring either one of the two conditions to allow the relabeling.

EXAMPLE 7 The set of owners consists of $o_{1}, o_{2}$ and $o_{3}$, unrelated by $\rho$, and the set of readers is $\left\{r_{1}, r_{2}, r_{3}\right\}$.

Define $L_{1}=\left\{o_{1}: r_{1}, r_{2} ; o_{2}: r_{1}, r_{3}\right\}$ and $L_{2}=\left\{o_{3}: r_{1}\right\}$. Then, by definition, $L_{1} \sqsubseteq L_{2}$ does not hold since there is no "acts for" relation between ob and either $o_{1}$ or $o_{2}$. But the only reader authorized by $o_{3}$ is also authorized by both $o_{1}$ and $o_{2}$ and so $U N\left(L_{2}\right) \subseteq I N T\left(C l_{\rho}\left(L_{1}\right)\right)$ holds.

The next one shows that the new condition would be too restrictive if used alone.

EXAMPLE 8 The set of owners consists of $o_{1}, o_{2}, o_{3}$ and $o_{4}$, with $\left(o_{3}, o_{1}\right) \in \rho$ and $\left(o_{3}, o_{2}\right) \in \rho$, and the set of unrelated readers is $\left\{r_{1}, r_{2}\right\}$. Define $L_{1}=$ $\left\{o_{1}: r_{1} ; o_{2}: r_{1}\right\}$ and $L_{2}=\left\{o_{3}: r_{1} ; o_{4}: r_{2}\right\}$. Then, by definition, $L_{1} \sqsubseteq L_{2}$ since $o_{3}$ acts for both $o_{1}$ and $o_{2}$, but $U N\left(L_{2}\right) \subseteq I N T\left(C l_{\rho}\left(L_{1}\right)\right)$ does not hold because of the policy of $\mathrm{O}_{4}$.

In the rest of this paper, we will write $C l(L)$ instead of $C l_{\rho}(L)$ when the hierarchy does not play a role or is implied by the context.

\section{$2.2 \quad$ Related Issues}

In this section, we discuss some of the issues relevant in the model to access files, databases and in general to perform I/O operations.

Consistency A first issue is the uniform and consistent treatment of files, I/O operations and/or channels. For example, in the model(s) presented by Myers and Liskov [6] the writing to an output channel is allowed only if the set of readers of the channel is a subset of the effective set of readers of the value written to the channel. In [5] the writing to a channel is allowed only if the label of the channel is more restrictive than the label of the value written to the channel. Now these definitions are not equivalent. For example, if a value label is $l(v)=\left\{o_{1}: r_{1}, r_{2} ; o_{2}: r_{1}\right\}$ and the channel label is $l(f)=\left\{o_{1}: r_{1}\right\}$ then, according to the first definition, writing the value $v$ on the channel $f$ is allowed, since $r_{1}$ is in the effective set of readers, while according to the second definition, writing is not allowed since the channel label is less restrictive (an owner was removed). Our approach is closer to the first definition. 
Declassification In [5] there are two concepts related to Declassification: the Authority predicate, and the Declassify operator. The Authority predicate specifies which principal can execute an application process and allows this correlation to be checked at run-time. Once the application process is running, then the explicit Declassify operator might be invoked with the desired label as a parameter. Since in [5], the Declassify operator can only remove policies which are owned by the principal listed in the Authority predicate, this check can be done at compile-time. No further check is done at run-time! This, however, presents a subtle problem with the files access and the I/O channels, as exemplified by the following taxpayer example from [5]. In this example, a tax-payer Bob likes to use a general tax program that accesses a proprietary database to which only the Preparer has access. The compiler does not allow writing back into Bob's file because the label was increased to include the Preparer. So the only way to allow this example to work, is to allow a declassification at run-time. In [5] the validity of the Declassify operation is checked at compile time, and since both the Authority predicate and the Declassify operator contain the explicit label "Preparer", the check succeeds.

This, however, raises two problems. First, this type of check limits the generality of the Declassify operator, since it requires that all parameters to Declassify and Authority be explicit at compile-time. Second, it also raises the problem of Covert channels, since anybody who can bypass the Authority predicate, can read the information from the Preparer database, can declassify it and can leak it via a covert channel. A more restrictive approach is desirable either at compile-time or at run-time.

The above issues call for a more precise definition of I/O operations and of the operation of declassification in the decentralized labels model, and represent the first goal of the present paper. The second goal is to extend the model to the database case.

\section{FILES AND I/O CHANNELS}

\subsection{Compile-time checks}

In the previous section, we mentioned two possible interpretations of permissions to write to a file and I/O operations. This section analyzes the issues and proposes our solution based on the intersection interpretation for writing. This solution for files and I/O channels are also extended to deal with administration, delegation, database operations and views in the following sections.

We assume that files have labels, so that a file $f$ has the label $l(f)$, and that $v$ is a variable with label $l(v)$, that is,

We define the permission to write to a file in terms of the two sets $\operatorname{INT}(L)$ and $U N(L)$, defined in Section 2. 
DEFINITION 9 (READ FROM A FILE) A read operation read $(f, v)$ of a file $f$ succeeds if $l(f) \sqsubseteq l(v)$,

that is, read $(\mathrm{f}, \mathrm{V})$ is allowed if the label $l(v)$ of the variable is at least as restrictive as the label $l(f)$ of the file.

DEFINITION 10 (WRITE TO A FILE) A write operation, write $(\mathrm{f}, \mathrm{V})$, to a file $f$ is allowed if $U N(l(f)) \subseteq I N T(C l(l(v)))$

that is write $(\mathrm{f}, \mathrm{V})$ succeeds if all the readers specified in the file label $l(f)$ are contained in the intersection of all the effective readers in the variable label $l(v)$.

EXAMPLE 11 (APPLICATION OF FILE RULES) If $l(v)=\left\{o_{1}: r_{1}, r_{2} ; o_{2}\right.$ : $\left.r_{1}\right\}$ and $l(f)=\left\{o_{2}: r_{1}\right\}$, then write $(\mathrm{f}, \mathrm{V})$ is granted, even though $l(f)$ is less restrictive than $l(v)$ since an owner is removed. Also, when $l(f)=\left\{o_{3}\right.$ : $\left.r_{1}\right\}$, the operation write $(\mathrm{f}, \mathrm{V})$ would be allowed.

Note that having on the file $f$ the label $\left\{o_{3}: r_{1}\right\}$ is useful in practice. Consider the two operations in sequence write $(\mathrm{f}, \mathrm{v})$, read $(\mathrm{f}, \mathrm{u})$, where label of $u$ is $\left\{o_{3}: r_{1}\right\}$. These operations appear equivalent to the assignment $\mathrm{u}$ $=\mathrm{v}$. However, the subsequent read from file $f$ into the variable $u$ with label $\left\{o_{3}: r_{1}\right\}$ is granted, even if $o_{3}$ is not an owner of $v$, while the assignment $u=$ $\mathrm{V}$ is denied since $l(v) \sqsubseteq l(u)$ does not hold.

If we allow the write $(\mathrm{f}, \mathrm{V})$ only to a file with at least as restrictive a label, the subsequent read in $u$ could not be performed, even if $\eta$ belonged to $I N T(C l(l(v)))$.

So far, all checks done at compile-time are similar to static type-checking: they make sure that the information flow along the valid paths is correct. In particular, the compilation of program Prog produces a set of constraints on the labels of the files and I/O channels that Prog can access. A run-time mechanism is needed to enforce that only the read and write operations on files and I/O channels that satisfy these constraints are allowed. Note that the runtime checks may be independent from the credential of the subject executing Prog. To clarify these concepts in the following we take a closer look at runtime checks.

\subsection{Run-time checks}

If all checks were made only at compile time and any subject could run the application, then the whole label structure could not guarantee the correct information flow. For example, if a subject logs into a system to run an application to read and write confidential data on a video-terminal, a run-time check is needed to compare the label of the I/O channels associated with the subject with the label of the input/output variables declared in the application. Therefore critical labels must be kept at run-time and some correlation must be 
checked between the subject running the application and the subjects appearing in the label of the variables.

A read operation read (IN, V) from an input channel $I N$ succeeds if $l(I N) \sqsubseteq l(v)$, that is, read (IN, $\mathrm{v})$ is allowed if the label $l(v)$ of the variable is at least as restrictive as the label $l(I N)$ of the Input channel. Note that the label $l(I N)$ may be determined by the authorization subsystem at run-time on the basis of the credentials of the subject associated with the channel IN (a typical example of such a channel is the subject's keyboard). Of course, also the label $l(v)$ of the variable where the data is read from $I N$ must be kept at run-time.

A write operation write (OUT.v) to the output channel OUT is allowed if $U N(l(O U T)) \subseteq I N T(C l(l(v)))$

Note that the label $l(O U T)$ may be determined at run-time according to the credentials of the subject which is associated with the channel OUT (a typical example of such a channel is a printer or a video-terminal).

Consider the sequence of the two operations read $(f, v)$; write (OUT, v) executed by a subject to display the content of a file $f$ on a video-terminal associated with the output channel OUT.

For the operation read $(\mathrm{f}, \mathrm{v})$ to be granted, we must have $l(f) \sqsubseteq l(v)$, which, by Propositions 2 and 5, implies $\operatorname{INT}(C l(l(v))) \subseteq I N T(C l(l(f)))$. If also the write (OUT, $v)$ is granted, then it must be $U N(l(O U T)) \subseteq I N T(C l(l(v)))$. Hence $U N(l(O U T)) \subseteq I N T(C l(l(f)))$ holds. This means that the union of all the readers in the label of the Output channel is contained in the intersection of the readers of file $f$; that is a subject that displays a file $f$ must be authorized by all the owners of $f$.

THEOREM 12 (SAFE INFORMATION FlOW) Under the conditions in Def. 6 , information flow from a file (channel) to another file (channel) is safe.

The proof is straightforward and will not be detailed here due to space limitations.

\subsection{The problem of Covert Channels}

In the discussion on $\mathrm{I} / \mathrm{O}$ channels above, we assumed that the program contains read or write operations to I/O channels, and the subject running the program is associated with the I/O channels accessed by the program and this correlation is checked at run-time.

A different situation occurs when the program performs only I/O operations on files. Who can execute such a program? What run-time checks are needed? In this situation, an illegal information flow outside the system may occur due to covert channels. 
In the following discussion, we assume that, from the point of view of the operating system, the subjects mentioned below (i.e. $\left.r_{1}, r_{2}, r_{3}, r_{4}\right)$ have the right to execute the program.

Consider a program $\operatorname{prog} R$ that executes the operation $\operatorname{read}(\mathrm{f}, \mathrm{v})$, that is, $\operatorname{prog} R$ attempts to read a file $f$, with label $l(f)=\left\{o_{1}: r_{1}, r_{2} ; o_{2}: r_{1}, r_{3}\right\}$ for example, into a local variable $v$. Subject $r_{1}$ should be allowed to execute the program and read $f$, since $r_{1}$ is authorized to read the file by all the owners.

Can a subject $r_{4}$ that does not appear in the label $l(f)$ be allowed to execute $\operatorname{prog} R$ ? Note that if we allow $r_{4}$ to execute read $(f, v)$ and read from file $f$ to $v, r_{4}$ cannot write $v$ into $r_{4}$ 's private files or other I/O channels because of the compile-time checks. However, $r_{4}$ could try to leak the contents of $v$ through a covert channel. For example, $r_{4}$ could design and execute a program ProgCC that first reads a sensitive file $f$ and then leaks its contents via a covert channel implemented by ProcCC itself. But, subject $r_{3}$ should be allowed to execute $\operatorname{prog} R$, since $r_{3}$ is in the policy of $o_{2}$. With such a restriction, the information that $r_{3}$ might leak is the one that $r_{3}$ is authorized to read, and could be leaked by $r_{3}$ anyway employing any other malicious covert channel; while without our restriction $r_{4}$ could leak the content of a file that $r_{4}$ is not authorized even to read. The above restriction must be enforced at run-time. It is worth noting the inevitability of covert channels whenever run-time checks are present. This problem arises because the run-time check may fail, and the fact of failure (or its absence) may lead to a leak of information via covert channel. Thus, limiting the class of users that can execute the program, reduces this risk of covert channels.

Consider another program $\operatorname{prog} W$ that executes the operation write $(f, v)$, that is $\operatorname{prog} W$ attempts to write into a file $f$. Should we check which subject can run $\operatorname{prog} W$ as well? This is not necessary, since the compile-time checks enforce the outwards information flow defined by the labels. Note, however, that one may employ additional access control policies, to limit the set of subjects who can write into a file. See also our discussion of Database access in Section 4.

To summarize this point, we check at run-time the subjects running an application for two purposes: 1) if the subject is not an owner, then limiting the execution only to subjects appearing in one of the label entries of the input channels has the advantage of restricting leakage via covert channels; 2) if the subject running the application is an owner, then some form of declassification is possible while keeping control over the dissemination of the information (see next section). 


\subsection{Declassification}

Our approach to declassification is that only owners of I/O channels and files can declassify information. This choice is inspired also by the originator controlled release model used by the DoD/Intelligence community.

The check on information declassification can be enforced both at compiletime or at run-time, based on the Authority predicate and the Declassify operator, discussed in Section 2. In addition, we want a principal specified in the authority predicate to be allowed to perform declassification only of information he owns. If the Authority predicate is employed to specify, in the code, the list of principals that can execute a program, then we can check at compile-time that every principal in that list is contained in the intersection of all the owners of all the files and input channels read by the program. If this is the case, then the Declassify operators present in the program are allowed, otherwise a compile-time error is returned. Of course, the compile-time check of declassification is quite restrictive, since not every execution will involve reading from all files. The difference between the above check and the check in [5] is that now the parameter to Declassify need not be explicit, thus the program may be more general (even though it involves more overhead...).

PROPOSITION 13 The "only owners" compile-time check is at least as restrictive as the check in [5].

The proof is direct. Every explicit principal in Declassify of [5] must have an owner policy, which means it is the owner of one of the read files, which is a necessary condition to being in the intersection of all owners of read files.

A less restrictive (and more precise) approach involves a run-time check. However, a run-time check requires to collect the information flow at run-time (similar to what is done by the Message filter approach of Jajodia et al [9]), and to check each invocation of a declassify operator $(D O)$ against the collected flow up to the invocation point of $D O$ (making sure that the principal executing the program is actually in the intersection of the set of owners of the current flow). Note that compared to the overhead in [9], there is less runtime overhead here, since we only need to store labels of files and input channels.

Summarizing, a compile-time approach restricts to the worst case the set of principals who can run a declassification program, while a run-time approach has a higher overhead, since it implies collecting the information flow and checking it for each declassify operator invoked.

The next example shows the interaction of the file operations and of the declassification issue in this model.

EXAMPle 14 (UPDATING A CUSTOMERS-ACCOUNT FILE) Consider a customer accounts file in a database and a customer who wants to read his account data, and update some personal information. The customer subject is 
$\mathrm{o}_{2}$ and the customer's private file is owned only by $\mathrm{o}_{2}$ and only $\mathrm{o}_{2}$ can read the file, that is, the private file is labeled by $\left\{o_{2}: r_{2}\right\}$. Similarly, the customers accounts file in the database is owned by the database administrator $\mathrm{q}$, and its label is $\left\{o_{1}: r_{1}\right\}$, so only $o_{1}$ can read/write to that file. Here are two scenarios (see Figure 1):

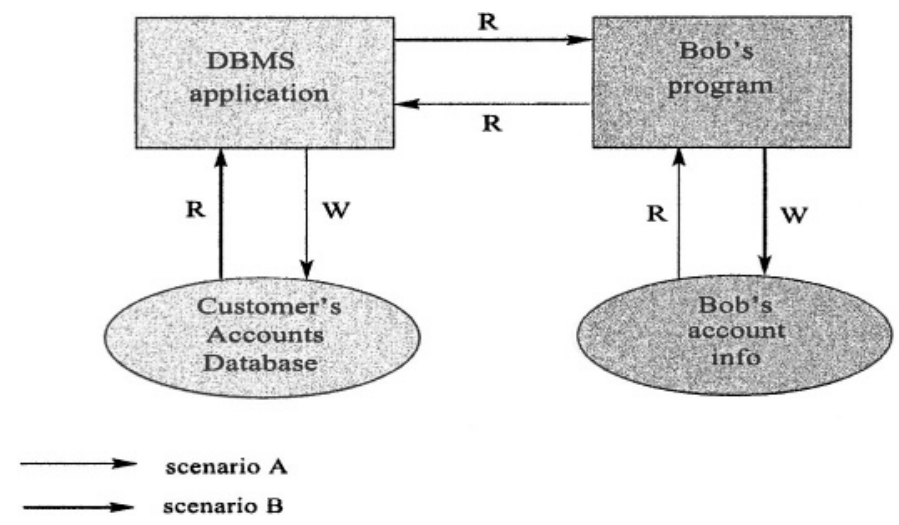

Figure 1. Accounts database example.

\section{Scenario A}

Owner $\mathrm{O}_{2}$ wants to update his account in the database with information from his private file. Then, $o_{2}$ reads his private file into a variable $p$ with label $\left\{_{2}\right.$ : $\left.r_{2}\right\}$, and variable $p$ is passed as a parameter to a program $Q W$ that updates the account data of $o_{2}$ into the customer account file. Program $Q W$ manages an array of slots V[n], each slot is labeled statically with label $\left\{a: r_{1} ; o_{i}: r_{i}\right\}$, where $o_{i}, r_{i}$ represents the $i$-th customer. QW compares the parameter label of $p$ with the label of the field V[j], using a switch or an if statement (like in the JFLOW language [4]), and sets $V[j]=p$ if the two labels agree. Now, assume that $V[j]$ has been updated on behalf of $o_{2}$, and has been labeled $\left\{o_{1}: r_{1} ; o_{2}\right.$ : $\left.r_{2}\right\}$, to allow the actual update of the database. Then, on should declassify V[j] and set it to $\left\{o_{1}: r_{1}\right\}$ only.

Next, the program $Q W$ invokes another program $Q$ ' with the privileges of $o_{1}$ (in the Unix system the program $Q$ ' could be implemented as a setuid-toprocess) and passes to $Q$ ' the parameter $V[j]$ to update the database. Since the label of V[j] now equals that of the customer accounts file owned by $\mathbf{q}$, the update of the database can be performed.

\section{Scenario $B$}

Owner $\boldsymbol{o}_{2}$ wants to read his account information from the database into a private file. Subject $o_{2}$ may call a program $Q R$ with setuid-to-o $o_{1}$, passing, as a parameter, a variable $p$ labeled $\left\{o_{2}: r_{2}\right\}$. QR can read the customer accounts database with $o_{1}$ privileges, and can enter the information into a $Q R$ local 
variable $x$ with label $\left\{o_{1}: r_{1} ; o_{2}: r_{2}\right\}$. Then, QR declassifies this information removing $o_{1}$ from the label of $x$, which becomes $\left\{o_{2}: r_{2}\right\}$. Program $Q R$ can do this since $Q R$ is running on behalf of the owner on of the database. Next, the program $Q R$ sets $p$ with the information readfrom $x$ and returns $p$ to $o_{2}$. $\mathrm{Now} \mathrm{O}_{2}$ can write the information back into his private file, since the label of $p$ is $\left\{o_{2}: r_{2}\right\}$.

The above two scenarios show that even under the restriction that only owners can declassify information, reads and updates of personal information into a shared database can be performed.

\subsection{Administration and Delegation}

In this section we discuss the policies to manage and administer file labels. Suppose a file has a label $\left\{o_{1}: r_{1}\right\}$. The following are possible administrative operations that can change labels of files:

1 Grant read - any listed owner can perform this operation, e.g., owner $q_{1}$ can change the label to $\left\{o_{1}: r_{1}, r_{2}\right\}$, granting read privileges to $r_{2}$.

2 Grant ownership - this operation adds an owner to the label. Any listed owner can perform this operation, e.g., owner $o_{1}$ can change the label to $\left\{o_{1}: r_{1}, r_{2} ; o_{2}: r_{2}\right\}$. Of course a more restrictive policy is also applicable, where only a specific owner (e.g. the super-user) can perform a grant ownership.

3 Revoke read access - any listed owner can perform this operation by deleting a reader from his list.

4 Revoke the ownership of another owner - in our model all owners are equal, so no owner can revoke another one, unlike the SQL database, where the Grant hierarchy is preserved.

5 Revoke self-ownership - we call this operation Administrative Declassification that any listed owner can perform by deleting himself from the owner field of the label.

3.5.1 Uses of administrative declassification. Administrative declassification is necessary to manage file labels. One interesting use of it is to deal with the problem of NON-originator controlled release. In an originator controlled release policy, the originator always maintains control on the information flow, even if it is copied to other files. This is the case also with the basic labels model, where the owners always maintain control. Now, sometimes an owner wants to have another user copy his file and to remove himself as responsible for this file. 
Suppose $o_{1}$ is the owner of $f 1$ with label $\left\{o_{1}: r_{1}\right\}$ and wants to give to $o_{2}$ a file $f 2$, a copy of $f 1$, without $o_{1}$ appearing as an owner of $f 2$. How can this be done in our model? There are two ways:

- Subject $o_{1}$ creates a file $f 2$ with both $o_{1}$ and $o_{2}$ as owners and adds $r_{2}$ as a reader in the label of $f 1$. Now subject $o_{2}$ can copy the file $f 1$ to $f 2$, since the label of $f 2$ is more restrictive than the label of $f 1$. Finally, $a$ removes himself as an owner of $f 2$ and removes $o_{2}$ as a reader from $f 1$.

- Subject $o_{2}$ creates a file $f 2$ with only $o_{2}$ as an owner. Subject $o_{1}$ adds $o_{2}$ as an owner for $f 1$ temporarily, and then $o_{1}$ reads file $f 1$ in a local variable, declassifies the data by removing his owner entry (i.e. the data are temporarily owned only by $o_{2}$ ) and writes it into $f 2$. Finally, $o_{2}$ uses administrative declassification to remove himself as an owner of file $f 1$.

Basically, the difference between the two scenarios is on who does the copying, but either way, $o_{\mathbf{1}}$ must be involved into resigning from the originator control on his data. That is, $\boldsymbol{o}_{1}$ cannot remove his own responsibility without performing an explicit operation.

\section{DATABASES AND VIEWS}

The decentralized labels approach can be extended to databases, both for compile time and run-time. A run-time information flow approach was investigated by Samarati et al. [9, 10]. A compile time approach for object-oriented databases was investigated by one of the authors in [8]. For this paper we do not distinguish between relational and object-oriented databases, but assume that all authorization information is associated with a View.

At this point we assume that a view has a single owner which can do the following:

1 grant access on the view to other users (read or write or define)

2 grant access with grant option. Users who get grant access can grant access to other users. Users who get both define and grant access can define new views based on their view and grant access to the new views to other users. A user who defines such a view becomes its owner.

In addition, we note the following:

- The view grant structure is known at compile time, and can be used by programs which use the view.

- The set of subjects who received read access on a view directly or indirectly is called the Closure of the view - $\operatorname{clos}(V)$. Obviously if view $V_{1}$ is derived from view $V_{0}$ then $\operatorname{clos}\left(V_{0}\right)$ contains $\operatorname{clos}\left(V_{1}\right)$. 
- Programs access the database by issuing a query on the view.

Since $\operatorname{clos}(V)$ is known at compile time, the query's label can be directly computed. However, since the query may not access all the attributes of the view, it is necessary to compute the closure of each accessible attribute separately. For an attribute $A$, we denote by $\operatorname{clos}(A, V)$ the closure of $A$ with respect to view $\mathrm{V}$ and define it as $\operatorname{clos}(A, V)=\left\{r: A \in V_{j}\right.$, $V_{j}$ derived from $V, r$ can read $\left.V_{j}\right\}$. That is, the closure includes all the users granted read access for the relevant attributes via the original view, or via any view derived from it.

In order to define the label of $A$ with respect to $\mathrm{V}$, we need to consider the owners of the different views which derive V. Therefore, we define the label of $A$ with respect to $\mathrm{V}$ as:

label $(A, V)=\left\{(o, r): A \in V, V_{j}\right.$ is $V$ or derives $V$, o $=$ owner $\left(V_{j}\right)$ and $\left.r \in \operatorname{clos}\left(A, V_{j}\right)\right\}$. This definition is justified as follows. Whenever we go up in the hierarchy, we are more restrictive, since we potentially add more owners, ( so the basic view will have only one owner in the label ). It is also justified by noting that revocation of rights in SQL databases is recursive.

To define the label of a query with respect to a view, we require that all the attributes that the query may access be defined in the view.

DEFINITION 15 (QUERY LABELS) The label of a query $Q$ which accesses attributes $A_{1}, \ldots A_{n}$ of a view $V$ is:

label $(Q, V)=\left\{(o, r): \forall i=1, \ldots, n A_{i} \in V_{j}, V_{j}\right.$ is $V$ or derived from $V$, $o=$ owner $\left(V_{j}\right)$ and $r \in I N T\left(\right.$ readers $\left.\left.\left(\operatorname{label}\left(A_{i}, V_{j}\right)\right)\right)\right\}$.

This label is defined as the set of owners of each of the derived views that contain all attributes accessed by the query, and the set of all the readers who have access to all the attributes appearing in the query (for that view).

Figure 2 illustrates this definition. The figure shows a view $V_{1}$ with attributes $A, B, C, D$, and two views $V_{2}$ and $V_{3}$ derived from it, and the readers who have access to each view. Assume that the owner of $V_{1}$ and $V_{3}$ is $o_{1}$, and the owner of $V_{2}$ is $o_{2}$, then

- the label of a query accessing attributes $A, B$ via the views $V_{2}$ and $V_{1}$ is $\{(o 1: r 1, r 2),(o 2: r 2)\}$ and $\{(o 1: r 1, r 2)\}$, respectively (i.e. the query accessing $V_{2}$ has a more restrictive label.)

- the label of a query accessing attributes $C, D$ via the views $V_{3}$ and $V_{1}$ is $\{o 1: r 3\}$ and $\{o 1: r 1, r 3\}$ respectively.

m the label of a query accessing attributes $A, C$ via the view $V_{1}$ is $\{o: r 1\}$

The above discussion defines how to compute the label $\operatorname{label}(Q, V)$ for a query $Q$ at compile-time. Using the above definitions one can define the no- 
tion of safe flow for databases similar to that of files, and apply both compiletime and run-time checks. We will not discuss it further here due to space limitations.

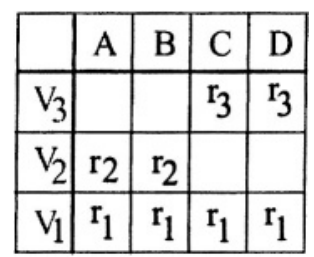

Figure 2. Example for Views labels.

\section{CONCLUSIONS}

Maintaining and enforcing the privacy and secrecy of data is recognized as a difficult problem, more so in an environment with decentralized control. Information flow control with the decentralized label model [6] is a step in the right direction. In the present paper, we have extended this work to deal in more details with files and I/O channels in section 3 and with databases and views in section 4 . In the process, we have argued that the rule $L_{2} \sqsubseteq L_{1}$ is too restrictive for many situations involving files and proposed more relaxed constraints that consider the readers to whom all the owners grant access.

Run-time checks are necessary to allow this form of controlled declassification. Though covert channels are possible, their impact can be limited by verifying the rights of the executors of a program.

Another issue is Administration. We have shown how various policies such as Delegation or Non-ORG control can be implemented using our model.

Among the several directions for future work is the investigation of alternative policies for declassification based, for example, on the presence of a given reader in the majority of the sets readers $(L, o)$, or in all the sets readers $(L, o)$ for a qualified subset of owners. Under investigation is also the problem of using the principal hierarchy to control the delegation, from an high level subject $S_{h}$ to a lower level subject $S_{l}$. For example, if a supervisor $S_{h}$ wants a technician $S_{l}$ to run a backup program on his behalf, with our run-time policy the technician cannot, unless $S_{l}$ is included in the label of the files. A useful extension of this policy would allow $S$ to run the backup if $S_{l}$ has an ancestor, with respect of the principal hierarchy, appearing as a reader in the label of the files.

Finally, we like to add that the definitions and proofs provided in this paper are quite intuitive. Our current research aims to develop a formal language and 
formal derivation rules on the labels, in the style of [6], to assist in the static analysis of the information flow.

\section{Acknowledgments}

This research was performed in part during a visit by the authors at the Center for Secure Information Systems at George Mason University, VA (USA).

\section{References}

[1] D. E. Bell and L. J. LaPadula. Secure Computer System: Unified Exposition and Multics Interpretation MTR-2997, MITRE Corp., Bedford, MA, March, 1976. reprinted in J. of Computer Security vol.4, no.2-3, pages 239-263, 1996.

[2] J. A. Goguen, and J. Meseguer. Security Policies and Security Models in Proc. 1982 IEEE Symposium on Security and Privacy, Oakland, CA, pages 11-20.

[3] J. McLean. Reasoning about Security Models, in Proc. 1987 IEEE Symposium on Security and Privacy, Oakland, CA, April 1987, pages 123-131. Also in Advances in Computer System Security, vol. III, ed. R. Turn, Artech House, Dedham, MA, 1988.

[4] A. C. Myers: JFlow: Practical Mostly-Static Information Flow Control. Proceedings, POPL 1999: 228-241

[5] A. C. Myers, and B. Liskov. Protecting Privacy using the Decentralized Label Model . Trans. on Software Engineering and Methodology, vol.9, no.4, October 2000, pages $410-442$.

[6] A. C. Myers, and B. Liskov. Complete, Safe Information Flow with Decentralized Labels. in Proc. IEEE Symposium on Security and Privacy, Oakland, CA, May 1998, pages 186197.

[7] R. Sandu and P. Samarati. Access Control: Principles and Practice. IEEE Communication Magazine, pages 40-48, 1994.

[8] M. Gendler and E. Gudes, "A compile-time Flow Analysis of Transactions and Methods in Object-oriented databases," Proceedings, 11 IFIP WG11.3 Database Security Conference, Lake Tahoe, CA. 1997.

[9] P. Samarati, E. Bertino, A. Ciampichetti and S. Jajodia "Information Flow Control in Object-Oriented Systems," IEEE Trans. on Knowledge and Data Engineering, July, 1997, 9(4), pages 524-539.

[10] P. Samarati, E. Bertino, A. Ciampichetti and S. Jajodia: Exception-Based Information Flow Control in Object-Oriented Systems. TISSEC 1(1), pages 26-65 (1998)

[11] S. Jajodia, R. Sandhu: Towards a Multilevel Secure Relational Data Model. Proceedings SIGMOD Conference 1991, pages 50-59

[12] S. Chen, D. Wijesekera, S. Jajodia: Flexflow: A flexible flow control policy specification framework Proceedings of IFIP WG11.3 Int. conference on data and application security, Estes park, Co., 2003.

[13] Yasuhiro Kirihata and Yoshiki Sameshima: A Web-based System for Prevention of Information Leakage proceedings of WWW2002, 2002. 